\title{
TRACHEOTOMY IN MAJOR CHEST INJURIES
}

BY

\author{
S. A. GUEUKDJIAN
}

From the Department of Surgery, Mayday Hospital, Croydon, Surrey

(RECEIVED FOR PUBLICATION MARCH 4, 1957)

Thoracic injuries, in particular fractures of the ribs, are very common industrial accidents. Most often these are of a mild nature and can be effectively treated in the works first-aid room or in casualty departments. Major crush injuries of the chest may seriously endanger life on account of involvement of the respiratory mechanism. Such accidents also present an urgent surgical problem; opinion is divided on the best method of treatment.

In severe injuries of the "stove-in" type involving fractures of several ribs anteriorly and posteriorly, the mortality rate is very high, seven out of eight cases, according to the Birmingham Accident Hospital statistics for 1944-52 (Proctor and London, 1955). Some time ago (Coroner's Reports, 1954) we lost a case of "steering wheel" injury of the thorax, and this in spite of every known method of treatment. Recently, however, in a patient suffering from severe thoracic fractures and respiratory embarrassment, emergency tracheotomy was performed and the patient's life was saved. The following case is therefore recorded.

\section{Case Report}

A miner, aged 38, was seen at the receiving room as an emergency after an accident in the pit. A derailed rolling wagon had crashed against him, pinning him against the tunnel wall. The patient was rescued by his companions, and after minor first-aid was rushed to hospital with crush injury of the chest.

On examination, the patient was shocked, restless, and moderately cyanotic. His breathing was troubled and noisy. There was an obvious paradoxical movement of the right antero-lateral half of the chest. Respiration rate was $\mathbf{4 0}$ per minute. Moist respiratory sounds were audible without auscultation. He had a small wound on the right hemithorax with considerable surgical emphysema and tension pneumothorax, obviously through valvular action. His pulse was rapid and the apex was deviated to the left. The jugular veins were distended but there was no subconjunctival haemorrhage or petechial exudate. Right pneumohaemothorax due to fractured ribs was diagnosed. Radiography confirmed the clinical impression of fractures of ribs $2-8$ on the right side anteriorly and revealed fractures of ribs 3-7 posteriorly on the same side. There was marked pneumothorax and a fluid level. No intra-abdominal injury was suspected.

It was urgently necessary to aspirate the right pleural cavity. Under local anaesthesia with $2 \%$ xylocaine, a Malecot self-retaining catheter was introduced by trocar and cannula through the fifth intercostal space. Considerable amounts of blood and air bubbles were obtained. The catheter was connected to a water-seal Winchestertype bottle placed on the floor. Mechanical suction was not needed as drainage seemed to be satisfactory. The right half of the chest was supported with zinc-oxide strapping. Penicillin was give prophylactically and the patient was nursed in a sitting position.

Eight hours after pleural closed drainage, the patient became distressed and showed signs of further respiratory obstruction. The chest was "bubbly" and tracheobronchial aspiration was contemplated. However, it was decided that tracheotomy would be of greater help. With the patient in a supine position on the operating table, which was tilted into the extreme reverse-Trendelenburg position, tracheotomy was performed under local anaesthesia. Through a vertical midline incision, the isthmus of the thyroid was separated between two clamps; the trachea was opened between the second and third rings and a double tracheotomy tube was inserted. On aspirating thick mucoid and slightly blood-stained tracheo-bronchial secretions with a suction catheter, the patient's distress visibly diminished. He was sent back to the ward and nursed in the sitting position. Atropine, grain $1 / 100$, was given and the patient had intermittent aerosol inhalations. Tracheo-bronchial aspiration was carried out at regular intervals and oxygen was made available in case of emergency.

By the following day, the cyanosis had disappeared, respiration was easier, and the chest excursions were less unequal. Improvement continued steadily. On the eighth day, radiography having shown considerable re-expansion of the lung, the pleural drain was removed and the wound closed. Two weeks after it was inserted, the tracheotomy tube was also removed. Productive expectoration was still present but this and the dyspnoea subsided slowly and serial radiographs showed a gradual return of normal lung fields. The rib fractures were also improving satisfactorily.

The patient was discharged symptom-free 21 days after admission to hospital, and at subsequent follow-up 
examinations his condition was satisfactory. He was given lighter work at the mine but felt that he could go back to his previous job.

\section{Discussion}

Although in the field of post-operative chest complications prophylactic tracheotomy has been advocated by many authors (Colvin and Morrison, 1953 , and others), its use in severe chest injuries is not common. Credit goes to Carter and Giuseffi (1953) who have demonstrated the value of emergency tracheotomy in such conditions.

The usual methods of strapping, rest, skeletal traction applied to the ribs or the sternum (Bailey and Matheson, 1953, and others), pericostal traction (Williams, 1948), immobilization in a plaster-ofParis cast (Belsey, 1953), open reduction (McKim, 1943; Sweet, 1954), and wiring (Coleman and Coleman, 1950) do not always give satisfactory results. In major chest injuries the primary cause for alarm is the tracheobronchial involvement. In such cases reduction of the fractures may be quite secondary as the respiratory condition demands more urgent attention.

Watson-Jones (1955) warns that an artificial respirator is sometimes needed. Gray (1942) and Hagen (1945) also advise the use of mechanical respirators. We have elsewhere (Lodge and Gueukdjian, 1955) stressed the importance of keeping the air passages free in cases of multiple chest injuries and have advocated emergency tracheotomy whenever asphyxia is to be feared.

The advantages of tracheotomy are that it is easy to perform under local anaesthesia. Although it is desirable to do the operation under surgical conditions, it can, in dire emergency, be done in heroic situations, necessitating few or no instruments. A pen-knife, stethoscope tubing, or a cut metal thermometer case may be useful in emergency (Lodge and Gueukdjian, 1955). Once inserted, a tracheotomy tube is easy to look after. Aspirations are simpler and more efficient than without the tube; the technique of aspiration can quickly be learnt by untrained staff. Tracheo-bronchoscopic aspiration is tiresome and exhausting for the patient, especially if repeated often. After tracheotomy, respiratory movements are more free. There is less tendency for the injured thoracic segment to "float" during inspiration and expiration (Carter and Giuseffi, 1951). Increased respiratory movements and reduction in the dead air-space after tracheotomy induce an increase in the effective tidal volume. As respiratory movements require less muscular exertion after tracheotomy, oxygen consumption is reduced.

There are, therefore, physiological as well as mechanical factors which render tracheotomy valuable in the emergency management of severe chest injuries.

\section{Summary}

After road accidents, industrial hazards account for the greatest number of serious crush-injuries of the chest. In cases of multiple rib fractures with damage to the respiratory mechanism, ventilation may be dangerously jeopardized. It is suggested that emergency tracheotomy can be of great help in the management of these cases. An illustrative case is reported.

\section{Addendum}

As this paper was going to press, my attention was drawn to an interesting report by Hulman (1957) on the successful use of tracheotomy for flail chest.

\section{REFERENCES}

Bailey, H., and Matheson, N. M. (1953). Emergency Surgery, 6th ed., p. 573. Wright, Bristol.

Belsey, R. (1953). Practitioner, 170, 134.

Carter, B. N., and Giuseffi, J. (1951). J. thorac. Surg., 21, 495.

Cont (1953). Surg. Gynec. Obstet., 96, 55.

Coleman, F. P., and Coleman, C. L. (1950). Ibid., 90, 129.

Colvin, E. M., and Morrison, W. M. (1953). Ibid., 96, 338.

Gray, H. K. (1942). Proc. Mayo Clin., 17, 566.

Gray, H. K.(1942). Proc. Mayo Clin., 17, 566.

Hagen, K. (1945). J. Bone Jt Surg.
Hulman, S. (1957). Lancet, 1, 454.

Lodge, W. O., and Gueukdjian, S. A. (1955). Practitioner, 175, 707.

Lodge, W. O., and Gueukdjian, S. A. (1955).

McKim, L. H. (1943). Ann. Surg., 118, 158. J. Surg., 42, 622

Proctor, H., and London, P. S. (1955). Brit. J. Surg., 42, 622.
Sweet, R. H. (1954). Thoracic Surgery, 2nd ed., p. 133. Saunders, Watsoniladelphia and London.

Watson-Jones, R. (1955). Fractures and Joint Injuries, 4th ed., vol. 2. p. 928 . Livingstone, Edinburgh.

Williams, M. H. (1948). Ann. Surg., 128, 1006. 